\title{
基于共同犯罪的犯罪人地域关系网络的 空间演化及其影响因素 以北京市诈骗案件为例
}

\author{
朱冠宇, 陈 鹏*
}

(中国人民公安大学警务信息工程与网络安全学院,北京 100038)

\begin{abstract}
摘 要: 当前,学界从犯罪人之间的关系模式、关系结构等方面对共同犯罪现象进行了一系列的研究,但缺少对犯罪 人地域关系的稳定性分析。论文根据北京市 2005 、2010、2014年街头诈骗案件数据,利用社会网络原理构建了基 于共同犯罪的犯罪人地域关系网络模型, 并利用网络分析等方法对参与共同犯罪的犯罪人地域关系网络的结构特 征及其变化趋势进行了分析。结果表明:在 2005-2014年间,参与北京市共同犯罪的犯罪人籍贯地域的空间分布 逐渐集中, 并形成了以华北地区为主、多个地域中心并存的格局; 犯罪人的地域关系网络小世界效应逐渐增强,并 从幂律分布模式向指数分布模式发展; 参与共同犯罪的犯罪人群中, 北京籍犯罪人的影响力逐渐下降, 而河北籍犯 罪人的影响力逐渐上升; 犯罪人地域关系网络的凝聚子群结构逐渐发生两极分化, 出现了少数共同犯罪关系异常 密切的犯罪人地域子群结构。针对犯罪人地域关系网络形成及演化的原因, 论文从外来人口社会关系重构与亚文 化的角度进行了分析和解释,对进一步开展犯罪人的共同犯罪关系模式研究具有一定的启示。
\end{abstract}

关 键 词: 诈骗案件; 地域关系网络; 对比分析; 社会网络分析; 北京

伴随着城市化的快速发展,城市中的社会问题 日趋增多, 尤其是治安和犯罪问题。长期以来,人 们对城市化过程中的犯罪现象给予了高度关注, 特 别是共同犯罪现象引起了人们的研究兴趣 ${ }^{[-4]}$ 。所 谓共同犯罪是指犯罪人之间通过分工合作的方式 进行犯罪的现象, 对这一现象, 学界从城市化过程 中人口的社会关系变化角度进行了解释, 其中代表 性的理论主要为亚文化理论 ${ }^{[5]}$ 。该理论认为随着城
市外来群体的大量增加,原有的本地化社会结构和 社会关系被打破,城市的文化多元性加强, 而相对 于原有的城市主文化,外来人群的地域性文化则属 于亚文化范围。因此, 为了更好地融人城市生活, 外来群体在一些行为上会更加注重团体性, 表现在 犯罪行为上主要为共同犯罪现象。

在参与共同犯罪的犯罪人关系结构上, 国外研 究人员进行了一系列的研究, 发现参与共同犯罪的

收稿日期: 2019-10-01; 修订日期: 2019-12-03。

基金项目: 北京市自然科学基金项目(9192022); 社会安全风险感知与防控大数据应用国家工程实验室主任基金项目; 中国人 民公安大学基本科研业务费课题(2018JKF228); 中国人民公安大学 2019 年拔尖人才培养专项资助硕士研究生科研 创新项目(2019ssky002)。[Foundation: National Natural Science Foundation of Beijing, No. 9192022; National Engineering Laboratory Director Fund for Social Security Risk Perception and Prevention and Control of Large Data Applications; Subject of Fundamental Research Business Fees of People's Public Security University of China, No. 2018JKF228; Top Talents Training Specialized Subsidy for Scientific Research and Innovation Projects of Master's Graduates of People's Public Security of China in 2019, No. 2019ssky002. ]

第一作者简介:朱冠宇(1996-), 男,山东苯庄人,硕士生,主要从事犯罪地理、社会网络分析方面的研究。

E-mail: 147288487@qq.com

*通信作者简介: 陈鹏(1981-), 男,辽宁营口人,博士, 副教授, 主要从事犯罪地理研究、犯罪情报分析等工作。

E-mail: chenpeng@ppsuc.edu.cn

引用格式: 朱冠宇, 陈鹏. 基于共同犯罪的犯罪人地域关系网络的空间演化及其影响因素: 以北京市诈骗案件为例 [J]. 地理科学进展, 2020, 39(5): 792-803. [Zhu Guanyu, Chen Peng. Spatial evolution and influencing factors of criminals' cross-area co-offending network: An example of fraud in Beijing. Progress in Geography, 2020, 39(5): 792-803. ] DOI: 10.18306/dlkxjz.2020.05.008 
特定犯罪人的关系构成会随着参与合作的犯罪人 年龄、犯罪类型等发生变化, 但参与共同犯罪的犯 罪人的整体关系模式会相对稳定 ${ }^{[6-8]}$ 。在共同犯罪 的整体关系模式上, 研究发现在很多共同犯罪案件 中,相同或相近的社会属性是其共同犯罪的基础, 尤其是同年龄、同性别、同家族、同民族、商业组织 归属相同或者籍贯地域相近的犯罪人之间更容易 形成共同犯罪关系 ${ }^{[9-16]}$ 。例如, Zhang 等 ${ }^{[17]}$ 发现在走 私网络中, 亲属关系与民族关系在关联处于不同分 销阶段的走私者方面起着重要作用; Charette 等 ${ }^{[16]}$ 运 用比例风险模型分析了芝加哥市 2006-2013 年间 的共同犯罪数据, 发现年龄、种族、性别、地理邻近 性和帮派身份方面的同质性能够导致持续的共同 犯罪关系的形成。此外,一些共同犯罪群体还包含 了来自不同家族、不同民族、不同籍贯地或不同商 业组织的犯罪人 ${ }^{[18]}$ 。例如, 与美国参议院调查的一 宗毒品贸易案件有关的交易网络中包含了 400 多名 来自不同文化、宗教和民族的犯罪人 ${ }^{[19]}$; Tenti 等 ${ }^{[20]}$ 对意大利毒品分销网络的分析也表明, 不同的犯罪 集团之间也会存在犯罪合作与依存关系。有证据 显示, 共同犯罪才伙往往属于机会驱动、整合个体 多种技能的临时型组织。因此, 有学者认为在一定 程度上突破民族、籍贯、家族、集团等关系的界限能 够使共同犯罪团伙得以适应不断变化的环境 ${ }^{[21]}$ 。 此外, 一些研究人员也指出, 同质性与异质性均是 共同犯罪团伙的重要特征, 人们往往关注其同质性 特点, 但仅对其同质性进行分析可能会导致无效的 犯罪控制措施 ${ }^{[22]}$ 。

在国内相关领域, 虽然有不少学者对外来人口 的社会关系结构及其特征给予了关注并开展了相 应的分析研究, 但缺少对城市中犯罪人群体共同犯 罪现象的深人分析 ${ }^{[23-24]}$ 。2016 年, 陈鹏等 ${ }^{[25]}$ 针对北 京市盗窃电动自行车案件分析了基于共同犯罪的 犯罪人群地域关系结构, 利用社会网络分析方法研 究了参与共同犯罪的犯罪人群的地域关系特征, 发 现来自少数地域的犯罪人是参与北京市共同犯罪 的主要力量。随后, 在 2017 年, Chen 等进一步分析 了基于共同犯罪的北京市 2012-2014年间盗窃电 动自行车案件的犯罪人地域关系, 发现参与共同犯 罪的犯罪人群主要来自北京及其周边区域, 并且犯 罪人的籍贯地域越相近, 其参与共同犯罪的可能性 越强 ${ }^{[26]}$ 。从目前已开展的工作来看, 国外的研究工 作更多集中于共同犯罪中犯罪个体一般社会属性 的关系分析, 缺少对个体的空间属性特别是地域特
征的关系研究,而国内虽然针对外来犯罪群体的地 域属性研究了参与共同犯罪的犯罪人地域关系结 构, 但缺少对这种结构的稳定性和趋势性分析。实 际上, 受城市社会文化及空间环境的约束和影响, 犯罪人之间的共同犯罪模式往往会随着犯罪人群 体在城市内的生活、工作、出行等因素的影响而发 生改变,进而导致犯罪人之间参与共同犯罪的关系 结构发生相应的调整,包括关系的产生、异化、衰 退、消失等 ${ }^{[27-33]}$ 。因此, 从犯罪人的地域特征角度研 究其在共同犯罪中的地域关系及其演变趋势将有 助于深人理解共同犯罪中犯罪人之间关系的稳定 模式及其变化,进而对了解犯罪人群之间的社会关 系结构具有重要作用。

综上, 本文针对北京市计骗类案件中犯罪人之 间的共同犯罪现象开展研究,基于共同犯罪构建犯 罪人之间的地域关系网络, 并对其结构进行态势和 趋势的演变分析, 以期从中发现共同犯罪中的犯罪 人地域关系的变化趋势, 并对其进行分析解释, 以 进一步丰富犯罪地理的研究。

\section{1 研究设计}

\section{1 数据来源}

本文研究数据收集自北京市公安局 2005、2010 与 2014 年发生的已破获街头诈骗案件信息。街头 计骗案件主要是指犯罪人编造理由,在街面、广场、 车站等地骗取受害人手机、银行卡、现金等财物的 犯罪活动。这类案件中犯罪人通常结伙作案, 分工 明确, 隐蔽性和危害性较强 ${ }^{[34]}$ 。在收集的数据中, 2005、2010和 2014 年已破获案件数量分别为 12000 、25649 和 16247 条。案件记录中包含了发案 时间、案件编号、犯罪人身份等基本信息。其中,案 件编号为案件的唯一标识,若不同犯罪人对应的案 件编号相同, 则表明其在该案件中存在共同犯罪关 系, 即隶属于同一个犯罪团伙。对犯罪人身份进行 地址解析则可以获取其原始的籍贯地址登记信息 (精确到地级市)。根据犯罪人的籍贯地信息以及犯 罪人的共同犯罪标签便可构建犯罪人的地域关系 网络。经数据清洗、篮选后最终得到 2005 年犯罪人 数量为 2033 人,来自 238 个地市; 2010 年犯罪人数 量为 3435 人, 来自 270 个地市; 2014 年犯罪人数量 为 2156 人,来自 254 个地市。

\section{2 基于共同犯罪的犯罪人地域关系网络模型构建} 共同犯罪是指由 2 个以上的犯罪人组成团伙组 
织共同策划并实施犯罪的行为。根据犯罪团伙成 员的籍贯地是否相同, 可以将其分为同地域共同犯 罪与跨地域共同犯罪。一般来说, 同地域共同犯罪 表现出的是犯罪人的地域集中性特征, 而跨地域共 同犯罪则反映了不同地域的犯罪人之间的沟通与 交流机制 ${ }^{[26]}$ 。为研究共同犯罪人地域属性中的异 质性特征, 引人社会网络概念, 建立基于共同犯罪 的犯罪人地域关系网络模型。将参与共同犯罪的 来自不同地域的犯罪人原始籍贯地抽象为网络节 点, 以 $V=\left\{v_{1}, v_{2}, v_{3}, \cdots, v_{z}\right\}$ 表示地域的集合, $Z$ 表示发 生跨地域共同犯罪关系的地域数量。设 $R=\left\{R_{1}\right.$, $\left.R_{2}, \cdots, R_{S}\right\}$ 为所有地域间关系的集合, $R_{i, j}$ 表示地域 $v_{i}$ 和地域 $v_{j}$ 之间的关系状态(即 2 个地域的犯罪人之 间是否存在跨地域共同犯罪关系)。若 $v_{i}$ 和 $v_{j}$ 之间 存在关系, 则 $R_{i, j} \neq \varnothing$ 且 $R_{i j} \subseteq R$; 若 $v_{i}$ 和 $v_{j}$ 之间不存在 关系, 则 $R_{i j}=\varnothing$ 。将 $v_{i}$ 和 $v_{j}$ 存在的关系抽象为网络的 边, 其矩阵表达式为:

$$
\begin{aligned}
& \boldsymbol{E}_{Z \times Z}=\left[\begin{array}{ccc}
e_{1,1} & \cdots & e_{1, z} \\
\vdots & & \vdots \\
e_{z, 1} & \cdots & e_{z, z}
\end{array}\right], \\
& \boldsymbol{e}_{i, j}=\left\{\begin{array}{ll}
n_{i, j}, & R_{i, j} \subseteq R, R_{i, j} \\
0, & R_{i, j}=\varnothing
\end{array} \quad(1 \leqslant i, j \leqslant Z)\right.
\end{aligned}
$$

式中: $n_{i, j}$ 代表来自地域 $v_{i}$ 和地域 $v_{j}$ 的犯罪人共同实 施犯罪的数量; $e_{i j}=n_{i j}$ 表示 $v_{i}$ 和 $v_{j}$ 之间存在边的连 接, 且连线权重为 $n_{i, j} ; e_{i, j}=0$ 表示 $v_{i}$ 和 $v_{j}$ 之间不存在边 的连接。

根据式(1), 可构建基于共同犯罪的 2005、2010 和 2014 年北京市街头计骗案件的犯罪人地域关系 网络结构(图 1)。

\section{2 研究方法}

\section{1 社会网络分析}

社会网络分析是一种用于对网络化数据结构 进行定量分析的数学方法, 其中衡量网络结构特征 的主要指标有网络密度、聚集系数、中心度、度分布 等(表 1)。

\section{2 凝聚子群分析}

凝聚子群是指在社会网络中存在的具有高度 凝聚力的 “子结构”, 通过凝聚子群分析可以得出社 会网络中存在的小团体 ${ }^{[35]}$ 。评价凝聚子群存在不 同的标准,其中较为常用的为派系分析。在一个无 向网络中, “派系”是指至少包含 3 个点的最大完备

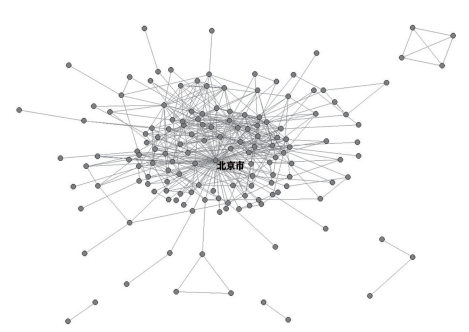

(a) 2005 年

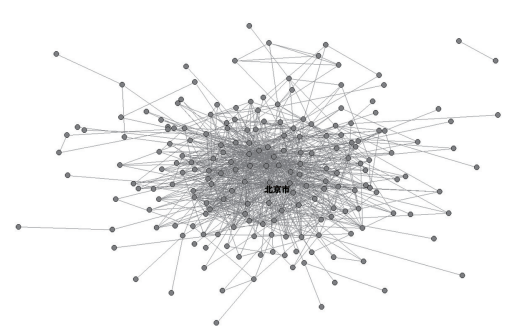

(b) 2010 年

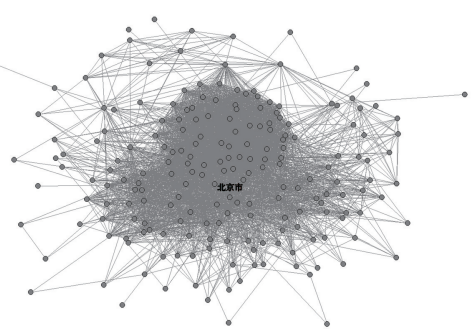

(c) 2014年

图 12005 、2010 和 2014 年北京市街头诈骗案件犯罪人跨地域关系网络拓扑结构

Fig.1 Topology of cross-area co-offending networks in 2005, 2010, and 2014

表 1 社会网络分析的主要特征指标

\begin{tabular}{|c|c|c|c|}
\hline 指标名称 & 表达式 & 表达式解释 & 参数解释 \\
\hline 网络密度 $(D)$ & $D=\frac{2 M}{Z(Z-1)}$ & 反映网络中节点之间关系的密集程度 & $\begin{array}{l}M \text { 表示网络中的边数, } Z \text { 为网络中节点 } \\
\text { 的数量 }\end{array}$ \\
\hline 聚集系数 $(C)$ & $C_{i}=\frac{2 E_{i}}{k_{i}\left(k_{i}-1\right)}$ & 反映图中节点之间聚集成团的程度 & $E_{i}$ 为 $k_{i}$ 个节点间相互连接的边数 \\
\hline 度数中心度 $\left(C_{D}\right)$ & $C_{D}(i)=\sum_{j=1}^{N} a_{i j}$ & 反映节点在网络中的重要性程度 & $\begin{array}{l}a_{i j} \text { 为表示社会网络关系的矩阵, 节点 } v_{i} \\
\text { 与 } v_{j} \text { 之间有关系则赋值为 } 1 \text {, 否则为 } 0\end{array}$ \\
\hline 中介中心度 $\left(C_{B}\right)$ & $C_{B}(i)=\sum_{j=1 ; k=1 ; j \neq k \neq i}^{N} \frac{N_{j k}(i)}{N_{j k}}$ & $\begin{array}{l}\text { 测量网络中所有最短路径中经过某一节点的 } \\
\text { 数量比例, 反映该节点对资源的控制程度 }\end{array}$ & $\begin{array}{l}N_{j k} \text { 表示节点 } v_{j} \text { 和 } v_{k} \text { 之间的最短路径条 } \\
\text { 数; } N_{j k}(i) \text { 表示节点 } v_{j} \text { 和 } v_{k} \text { 之间的最短路 } \\
\text { 径经过节点 } v_{i} \text { 的条数 }\end{array}$ \\
\hline 强度中心度 $\left(C_{S}\right)$ & $C_{S}(i)=\sum_{j \in N_{i}} w_{i j}$ & $\begin{array}{l}\text { 测量节点所有关联的关系的权重的集合, 反 } \\
\text { 映节点考虑连接关系权重的重要性程度 }\end{array}$ & $\begin{array}{l}N_{i} \text { 表示节点 } v_{i} \text { 的相邻节点集合; } w_{i j} \text { 表示 } \\
\text { 连接节点 } v_{i} \text { 和节点 } v_{j} \text { 的关系权重 }\end{array}$ \\
\hline
\end{tabular}

Tab.1 Main indicators of social network analysis 
子网络。这表明:一个派系至少包含 3 个点; 派系是 完备的,派系中任何 2 点之间都存在直接联系; 派系 是“最大”的, 即向这个子图中增加任何一点, 将改 变其“完备”的性质 ${ }^{[36]}$ 。本文将采用 “派系”这一概 念来对犯罪人跨地域共同犯罪关系网络中的凝聚 子群进行挖掘与分析。

实际上, 当派系的拓扑结构相同但内部节点关 联强度不同时, 派系的凝聚性与紧密性也有差异。 假设网络 $W$ 中存在一个派系子网络 $G=\left(V^{\prime}, E^{\prime}\right)$, 该 派系结构节点数为 $Z_{G}$, 则该子网络对应的邻接矩阵 可以表示为:

$$
\boldsymbol{E}_{Z_{G} \times Z_{G}}^{\prime}=\left[\begin{array}{ccc}
e_{1,1} & \cdots & e_{1, Z_{G}} \\
\vdots & & \vdots \\
e_{Z_{G}, 1} & \cdots & e_{Z_{G}, Z_{G}}
\end{array}\right], e_{i, j}=m_{i, j}\left(1 \leqslant i, j \leqslant Z_{G}, Z_{G} \geqslant 3\right)
$$

式中: $m_{i, j}$ 代表节点 $v_{i} 、 v_{j}$ 之间的关联强度。与一般网 络不同的是, 当 $i \neq j$ 时, 派系子网络的邻接矩阵元素 $e_{i, j} \neq 0$, 即派系中任意 2 点之间均存在连接。定义子 群凝聚系数 $T$ 为派系子网络邻接矩阵元素中的最小 值(不考虑矩阵主对角线元素), 即:

$$
T=\min \left|e_{i, j}\right|\left(1 \leqslant i, j \leqslant Z_{G}, i \neq j\right)
$$

则子群凝聚系数 $T$ 实际上就表示一个派系结构 中关联强度最弱的连线权重, 从而可以用来表征一 个派系的凝聚程度。

\section{3 结果分析}

\section{1 基于共同犯罪的犯罪人地域关系网络空间格局 分布}

在犯罪人的地域关系网络中, 节点代表犯罪人
的籍贯地,基于共同犯罪的犯罪人地域关系形成过 程实际上是来自不同籍贯地的犯罪人群之间合作 关系的确立过程。图 2为 2005、2010和 2014年参与 北京市街头计骗案件共同犯罪的犯罪人地域关系 网络节点的空间分布情况, 可见, 这期间的犯罪人 地域关系网络的空间格局发生了较大变化。首先, 参与北京市计骗类案件共同犯罪的犯罪人的籍贯 地分布更加紧凑。在 2005 和 2010 年, 共同犯罪人 包含了来自新疆喀什等地的成员, 地域范围基本遍 布全国,但在 2014 年,共同犯罪人不再包含来自新 疆等地的成员。其次, 犯罪合作关系出现在了更多 的地域之间,从图中可以看到, 2005 年和 2010 年不 同地域犯罪人之间的共同犯罪关系较为稀疏,但在 2014 年, 这种关系明显变得更加密集。第三, 从核 心的地域节点空间分布来看,基于共同犯罪的犯罪 人地域关系网络呈现出多热点的趋势。在 2005 年, 网络中具有较大连接度的节点主要集中在华北和 东北等地, 形成了双热点地域节点群; 在 2010 年, 东 北地区的节点连接度有所下降, 而华北地区则成为 了唯一的高节点连接度地区, 形成了单热点地域节 点群; 但是在 2014 年, 除华北地区节点的连接度进 一步增强外, 东北、西北和西南等地的节点连接度 也明显增大, 出现了多热点地域节点群并存的 现象。

\section{2 基于共同犯罪的犯罪人地域关系网络统计特征 分析}

3.2.1 北京市街头计骗案件犯罪人跨地域合作关系 更为紧密, 并呈现去中心化趋势

利用社会网络分析方法对 2005、2010和 2014 年北京市街头计骗案件中参与共同犯罪的犯罪人
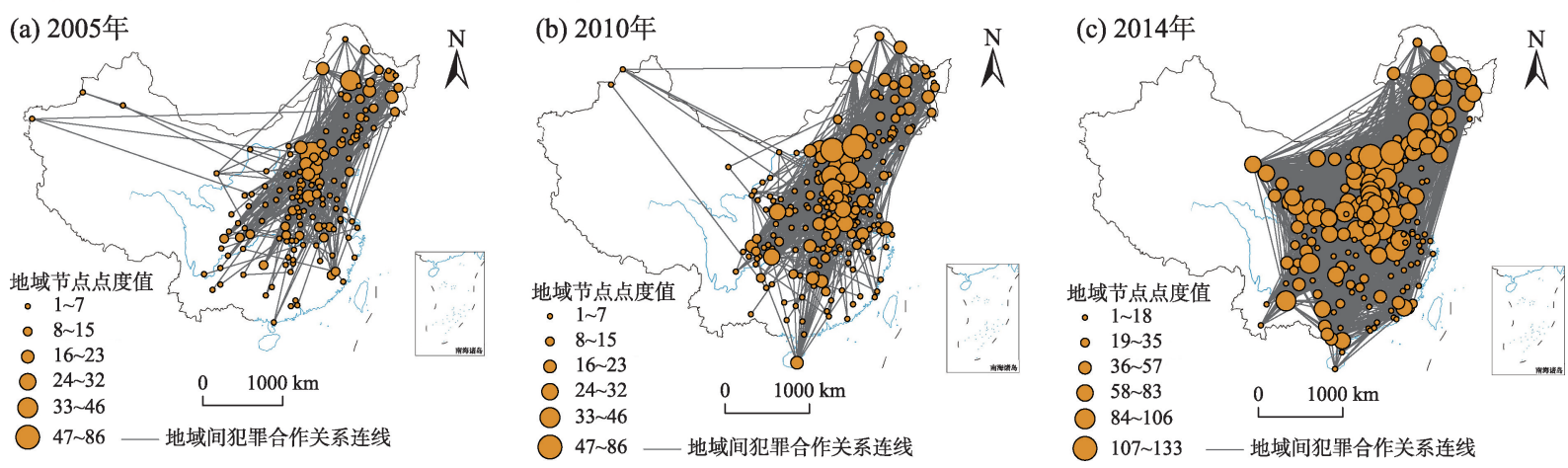

注: 本图基于自然资源部标准地图服务网站下载的审图号为 GS(2016)1569号的标准地图制作, 底图无修改。

图 2 基于共同犯罪的犯罪人地域关系网络的空间结构

Fig.2 Spatial structure of the cross-area co-offending networks 
地域关系网络结构进行分析,表 2 为主要指标的统 计结果。可见, 在网络密度方面, 网络在规模扩大的 同时, 其连线数 $(M)$ 、网络密度 $(D)$ 与平均度 ${ }^{\mathbb{1}}(A v g . D)$ 都有更大程度的提升, 网络变得更为密集, 连通程 度大大增强。其次, 在网络小世界性方面, 将不同 年份网络的聚集系数 $C_{1}$ 、同等规模随机网络的聚集 系数 $C_{2}$ 进行相互比较, 发现犯罪人地域关系网络具 有高聚集性, 且聚集性还在继续增强。此外, 虽然 2014 年犯罪人地域关系网络覆盖的地域节点数量 有所增加, 但网络的平均最短路径 $L$ 却大大减小。 从小世界效应强度 ${ }^{\left[{ }^{37}\right.}$ 来看, 与 2005 年相比, 2014 年 的犯罪人地域关系网络的小世界系数提升了 1.72 倍。以上结果表明, 来自各个地域犯罪人之间的共 同犯罪关系越来越紧密, 倾向于形成一个高效连通 的有机整体。

分别对基于共同犯罪的 2005-2014年北京市 街头诈骗案件犯罪人地域关系网络进行累积度分 布统计, 得到网络中各地域节点的累积度分布概率 $P(k)$, 如图 3 所示。结果发现, 2005 和 2010 年的犯 罪人地域网络具有一定的“拖尾”特征, 表明来自不 同地域的犯罪人与个别核心地域的犯罪人之间存 在着较为密切的犯罪合作关系。但在 2014 年, 网络 的结构特征则发生较大变化, 其累积度分布的不均 衡性已大大减弱, 来自更多地域的犯罪人开始拥有 更为广泛的跨地域犯罪合作关系。一般地, 随机网
络的累积度分布多表现为指数或对数分布; 而无标 度网络则体现出不均衡性与偏好性, 其累积度分布 表现为幂指数型 ${ }^{[38]}$ 。对 2005、2010 和 2014 年的网 络节点度累积分布分别进行函数拟合(表 3 ), 发现 2005 年网络的累积度分布 $P(k)$ 的幂次函数关系相 较指数关系而言更为明显 $\left(R^{2}=0.9013>0.6680\right), 2010$ 年则比较接近, 但幂次函数特征仍高于指数函数 $\left(R^{2}=0.8809>0.8668\right)$, 而 2014 年网络的幂次函数的 拟合度远小于指数函数 $\left(R^{2}=0.5741<0.8932\right)$ 。该结 果表明,该犯罪人地域关系网络的幂律特性在逐渐 减弱, 同时整体网络在向具有随机网络特征的指数 分布发展, 说明犯罪人的跨地域犯罪合作关系不再 受个别核心地域犯罪人群支配, 网络的中心化与集 中度有所下降。

3.2.2 北京籍犯罪人的影响力逐渐下降, 河北籍犯 罪人的影响力逐渐上升

在对犯罪人地域关系网络进行整体网络结构 特征分析的基础上,进一步利用社会网络分析中的 中心度指标对网络中具体节点的影响力特征进行 统计 ${ }^{[39]}$ 。表 4 分别给出了 2005、2010 和 2014 年参与 北京市共同犯罪的犯罪人地域关系网络中排名前 10 位的度数中心度、中介中心度、强度中心度的地 域节点。首先, 从度数中心度来看, 在 2005 年, 北京 处于犯罪人地域关系网络中的绝对核心地位, 网络 为单中心模式; 但在 2010 年,张家口、承德等地的度

表 2 基于共同犯罪的犯罪人地域关系网络整体网指标统计

Tab.2 Whole network indicator statistics of cross-area co-offending networks

\begin{tabular}{cccccccccc}
\hline 年份 & $Z$ & $M$ & $D$ & $A v g . D$ & $C_{1}$ & $C_{2}$ & $C_{1} / L$ \\
\hline 2005 & 151 & 485 & 0.0428 & 6.4238 & 0.6240 & 0.0444 & 0.2353 \\
2010 & 199 & 988 & 0.0501 & 9.9296 & 0.6000 & 0.0500 & 2.6296 \\
2014 & 203 & 4241 & 0.2068 & 41.7833 & 0.7936 & 0.2067 & 1.9652 \\
\hline
\end{tabular}

(a) 2005年

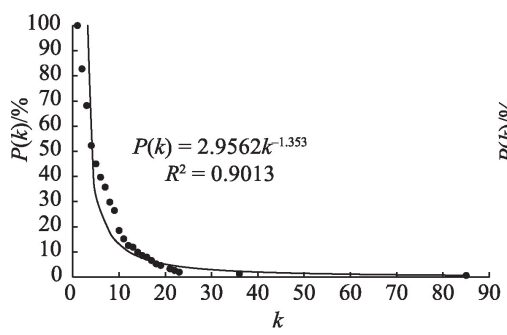

(b) 2010 年

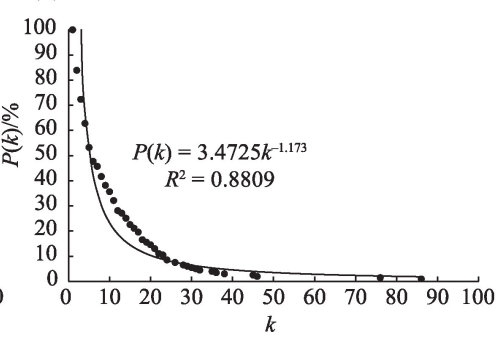

(c) 2014年

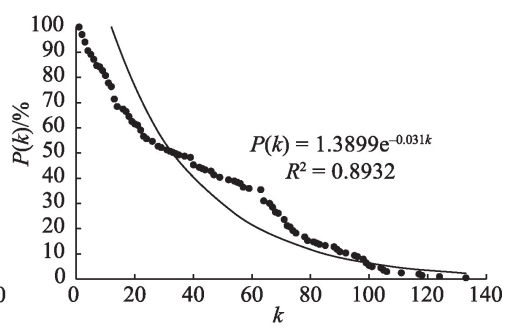

图 3 基于共同犯罪的犯罪人地域关系网络累积度分布概率

Fig.3 Cumulative degree distribution of cross-area co-offending networks

(1) 网络平均度是指所有节点度的平均值, 本文用 $A v g . D$ 来表示。 
表 3 基于共同犯罪的犯罪人地域关系网络 累积度分布函数拟合结果

Tab.3 Function fitting results of cumulative degree distribution of cross-area co-offending networks

\begin{tabular}{ccc}
\hline \multirow{2}{*}{ 年份 } & \multicolumn{2}{c}{ 拟合函数 } \\
\cline { 2 - 4 } & 幂次函数 & 指数函数 \\
\hline 2005 & $P(k)=2.9562 k^{-1.353}, R^{2}=0.9013$ & $P(k)=0.341 \mathrm{e}^{-0.066 k}, R^{2}=0.6680$ \\
2010 & $P(k)=3.4725 k^{-1.173}, R^{2}=0.8809$ & $P(k)=0.5329 \mathrm{e}^{-0.06 k}, R^{2}=0.8668$ \\
2014 & $P(k)=5.7008 k^{-0.849}, R^{2}=0.5741$ & $P(k)=1.3899 \mathrm{e}^{-0.031 k}, R^{2}=0.8932$ \\
\hline
\end{tabular}

数中心度增长显著, 与北京之间的差距缩小; 到了 2014 年, 北京则下降至第四位, 河北的保定、张家 口、承德上升为前 3 位, 表明来自这几个地区的犯罪 人在北京市共同犯罪地域关系网络中的影响力增 大。此外,从具体的地域节点分布上来看,与 2005 年和 2010 年相比, 2014 年网络中的高影响力犯罪 人籍贯地域节点的组成发生了较大的变化, 原先较 为重要的地域节点如牡丹江、哈尔滨、菏泽等地被 承德、徐州等地所取代。其次, 从中介中心性的分 布来看,在 2005-2014年犯罪人地域关系网络中， 北京的中介中心性始终最高,表明来自北京的犯罪 人在计骗类共同犯罪中扮演了最为核心的角色,但 同时其媒介作用在逐渐减弱。第三, 从强度中心度
来看, 2005 年北京的强度中心度为 1612 ,表明来自 北京的犯罪人与其他各地域犯罪人共同实施的诈 骗类犯罪活动次数高达 1612 次,但在 2010 年, 北京 市的强度中心度则被承德反超降至第二位,其指标 下降至 656 次, 而在 2014 年, 北京的强度中心度进 一步下降至 237 次, 本地犯罪人与其他各地域犯罪 人合伙作案次数之和进一步减少,而与此同时, 呼 伦贝尔、绵阳、廊坊、沧州、邯䣋、鹤岗等地域节点在 网络中的强度中心度则增长十分明显, 均超过了 1000 , 表明来自这几个地域的犯罪人与其他地域犯 罪人合作犯罪的频次大大增加。以上分析表明,在 2005 、2010 和 2014 年间, 参与北京市街头计骗案件 共同犯罪的犯罪人群中,北京籍犯罪人的影响力在 逐渐下降,而来自华北特别是河北地区的犯罪人的 影响力逐渐上升。

3.2.3 犯罪人地域关系网络子群结构发生两极分 化,出现了较强的核心地域群体

在基于共同犯罪的犯罪人地域关系网络中,除 重要的地域节点外, 由若干地域节点构成的凝聚性 子群结构也是一个值得关注的特征, 因为该结构一 般包含了不同地域犯罪人的共同犯罪关系模式。为 了发现参与北京市犯罪人地域关系网络中的关键子

表4 中心度前 10 位地域节点统计

Tab.4 Statistics of the top 10 regional nodes based on degree centrality

\begin{tabular}{|c|c|c|c|c|c|c|c|c|c|}
\hline \multirow{2}{*}{ 排序 } & \multicolumn{3}{|c|}{ 点度中心度 } & \multicolumn{3}{|c|}{ 中介中心度 } & \multicolumn{3}{|c|}{ 强度中心度 } \\
\hline & 2005 年 & 2010年 & 2014年 & 2005年 & 2010年 & 2014年 & 2005 年 & 2010年 & 2014年 \\
\hline \multirow[t]{2}{*}{1} & 北京 & 北京 & 保定 & 北京 & 北京 & 北京 & 北京 & 承德 & 呼伦贝尔 \\
\hline & (85) & (86) & (133) & $(0.543)$ & $(0.275)$ & $(0.068)$ & (1612) & (669) & (1378) \\
\hline \multirow[t]{2}{*}{2} & 齐齐哈尔 & 张家口 & 张家口 & 齐齐哈尔 & 张家口 & 保定 & 衡水 & 北京 & 绵阳 \\
\hline & $(36)$ & (86) & (124) & $(0.064)$ & $(0.226)$ & $(0.055)$ & (879) & (656) & (1338) \\
\hline \multirow[t]{2}{*}{3} & 沧州 & 承德 & 承德 & 牡丹江 & 承德 & 咸阳 & 齐齐哈尔 & 保定 & 廊坊 \\
\hline & (23) & (76) & (118) & $(0.052)$ & $(0.105)$ & $(0.042)$ & (870) & (606) & (1297) \\
\hline \multirow[t]{2}{*}{4} & 牡丹江 & 石家庄 & 北京 & 沧州 & 石家庄 & 张家口 & 德州 & 张家口 & 沧州 \\
\hline & (22) & (46) & (117) & $(0.048)$ & $(0.062)$ & $(0.038)$ & (862) & (556) & (1271) \\
\hline \multirow[t]{2}{*}{5} & 哈尔滨 & 邯䣋 & 齐齐哈尔 & 衡水 & 保定 & 徐州 & 呼伦贝尔 & 聊城 & 期䣋 \\
\hline & $(21)$ & (45) & (111) & $(0.043)$ & $(0.048)$ & $(0.036)$ & (733) & $(465)$ & (1244) \\
\hline \multirow[t]{2}{*}{6} & 菏泽 & 保定 & 徐州 & 沈阳 & 沧州 & 湛江 & 延边 & 沧州 & 鹤岗 \\
\hline & (19) & (38) & (106) & $(0.040)$ & $(0.044)$ & $(0.035)$ & (700) & (439) & (1212) \\
\hline \multirow[t]{2}{*}{7} & 保定 & 沧州 & 济宁 & 南平 & 达州 & 齐齐哈尔 & 南平 & 潜江 & 保定 \\
\hline & (19) & (36) & (105) & $(0.026)$ & $(0.042)$ & $(0.032)$ & (700) & $(342)$ & (270) \\
\hline \multirow[t]{2}{*}{8} & 衡水 & 德州 & 沧州 & 太原 & 菏泽 & 廊坊 & 秦皇岛 & 宜昌 & 北京 \\
\hline & (18) & (35) & (104) & $(0.025)$ & $(0.041)$ & $(0.024)$ & (301) & (331) & (237) \\
\hline \multirow[t]{2}{*}{9} & 呼伦贝尔 & 信阳 & 邢台 & 广州 & 信阳 & 河池 & 保定 & 牡丹江 & 张家口 \\
\hline & (17) & $(32)$ & (104) & $(0.025)$ & $(0.041)$ & $(0.024)$ & (259) & (316) & (229) \\
\hline \multirow[t]{2}{*}{10} & 张家口 & 重庆 & 咸阳 & 邯䣋 & 六安 & 承德 & 咸宁 & 德州 & 齐齐哈尔 \\
\hline & (17) & (31) & (101) & $(0.024)$ & $(0.036)$ & $(0.024)$ & (225) & (306) & $(225)$ \\
\hline
\end{tabular}


群结构及变化情况, 本文提出子群检测策略来进行 网络的凝聚子群分析, 其具体实施步骤如下所示 ${ }^{[00]}$ :

(1) 设定 $t=1$;

(2) 在网络 $W$ 中, 若 $n_{i, j}<t(1 \leqslant i, j \leqslant Z)$, 则将该连 线删除, 形成子网络 $W_{t}$;

(3) 对子网络 $W_{t}$ 进行派系分析, 得到其凝聚子 群数量 $N_{t}$ 和每个子群具体的节点组成;

(4) 记录本次子群检测结果 $\left(t, N_{t}\right)$ 。该结果表 明, 在网络 $W$ 中子群凝聚系数 $T \geqslant t$ 的凝聚子群数量 为 $N_{t}$;

(5) 设定 $t=t+1$, 并重复步骤(2)至(5), 直至 $N_{t}=0$ 。 本文采用 “派系”这一概念对凝聚子群进行界 定, 并利用该子群检测策略分别对 2005-2014年犯 罪人的地域关系网络进行凝聚子群分析, 并将每一 步骤的子群检测结果 $\left(t, N_{t}\right)$ 绘制为折线图(图 4)。

可见, 在 2005、2010和2014 年间, 犯罪人地域 关系网络的子群结构特征发生了较大改变。在 2005 年, 网络中 $T \geqslant 1$ 的凝聚子群数量为 102 , 之后随 着 $t$ 值的增大, 网络凝聚子群数量下降较为缓慢; 当 $T \geqslant 61$ 时, 网络中凝聚子群数量下降为 2 。2010年网 络的凝聚子群结构与 2005 年较为相似, 当 $T \geqslant 45$ 时, 网络中凝聚子群数量下降为 2 。而在 2014 年, 网络 中 $T \geqslant 1$ 的凝聚子群数量多达 835 个, 明显大于 2005 年和 2010 年, 之后随着 $t$ 值的增大, 网络的凝聚子群 数量迅速下降; 当 $T \geqslant 14$ 时凝聚子群数量便下降为 2 , 当 $T \geqslant 15$ 时网络中凝聚子群数量仅为 1 。这一结 果反映出在 2005-2010 年间犯罪人地域关系网络 中凝聚子群的数量较少, 共同犯罪现象主要存在于 两两地域的犯罪人群之间。但在 2014 年, 犯罪人地

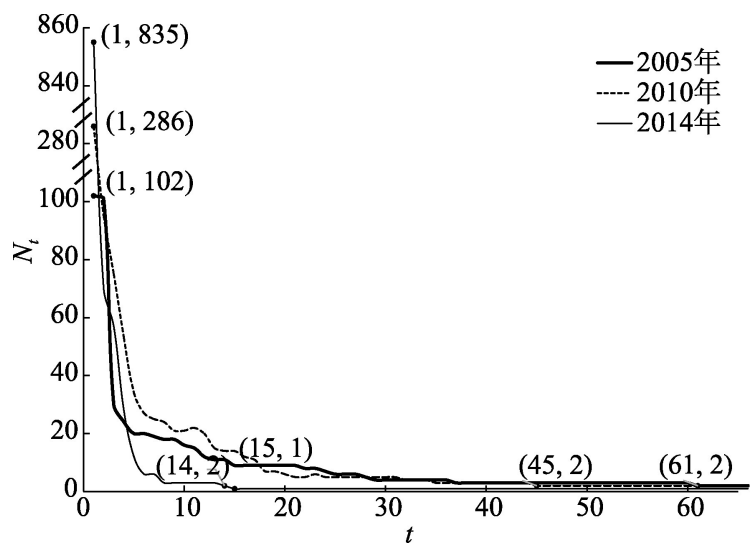

图 4 犯罪人跨地域共同犯罪关系网络子群检测结果

Fig.4 Cohesive subgroup detection results of cross-area co-offending networks
域关系网络中出现了更多的凝聚子群结构, 表明有 多个地域的犯罪人参与了共同犯罪,共同犯罪人的 地域背景呈现出多元化和复杂化趋势, 但子群的凝 聚性普遍降低,不同地域犯罪人之间组合的随机性 增强。图 5 所示分别为 2005 、2010 和 2014年高凝聚 子群结构节点的空间分布, 其中 2005 年犯罪人地域 关系网络中凝聚度最高的 2 个凝聚子群分别由北 京、秦皇岛、咸宁 3 个节点与呼伦贝尔、齐齐哈尔、北 京、衡水、德州、南平、延边 7 个节点组成, 子群内节 点之间的最低共同犯罪关系强度分别为 108 与 115 (图 5a、5b)。而在 2010 年, 最为显著的 2 个凝聚子群 结构分别由巴彦卓尔、运城、阳泉和承德、衡水及牡 丹江构成, 子群内节点之间的最低共同犯罪关系强 度仅为 86 和 93(图 5c、5d)。然而在 2014年, 凝聚度 最高的 2 个凝聚子群分别由齐齐哈尔、锦州、抚州 3 个节点与呼伦贝尔、鹤岗、廊坊、沧州、邯䣋、绵阳 6 个节点组成, 其中前一子群内节点之间的最低合作 关系强度仅为 14(图 5e), 但后一子群内节点之间的 最低合作强度则达到了225(图 5f)。该现象表明,在 2005-2010年的北京市街头计骗类案件中, 不同地 域的犯罪人之间的共同犯罪联系程度较为紧密、合 作关系较为均衡, 地域组合具有一定的稳定性。但 是在 2014 年, 不同地域犯罪人之间的共同犯罪关系 发生两极分化, 大多数地域犯罪子群的凝聚程度降 低, 同时也出现了少数共同犯罪合作关系异常密切 的地域子群结构, 反映出参与共同犯罪的犯罪人核 心地域开始形成。

\section{3 基于共同犯罪的犯罪人地域关系网络的形成原} 因分析

基于共同犯罪的犯罪人地域关系网络实际上 是分布于北京市来自于不同地域的外来人口之间 互相拉拢合作形成大量共同犯罪案件的中观表 现。近年来, 随着中国城市化进程的日益加快, 大 量人口不断涌人发达城市, 形成了城市间规模巨大 的人口迁徙和流动现象, 由此带来了外来人口融人 城市问题 ${ }^{[4]}$ 。从本质上, 外来人口融人城市其实是 外来人口通过在新的社会环境下重建自己的社会 关系来实现自身的空间、身份和价值融人的过程。 外来人口在进人新的环境后, 其通过自身的社会参 与获取到了新的社会资源, 社会关系结构发生较大 改变, 其主要表现为原有的乡土社会关系逐渐弱 化,而在新的城市环境下形成了新的邻里关系、社 会关系、同事关系, 从而实现了自身地缘、业缘关系 


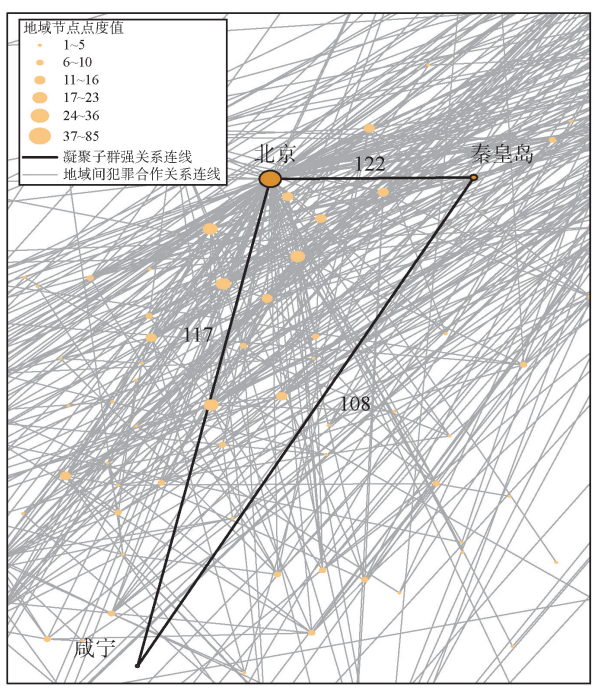

(a) 2005年子群一

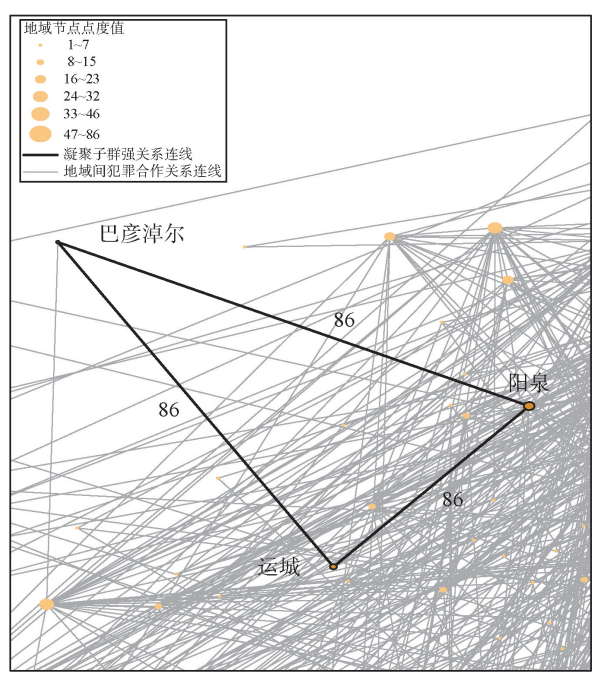

(c) 2010年子群一

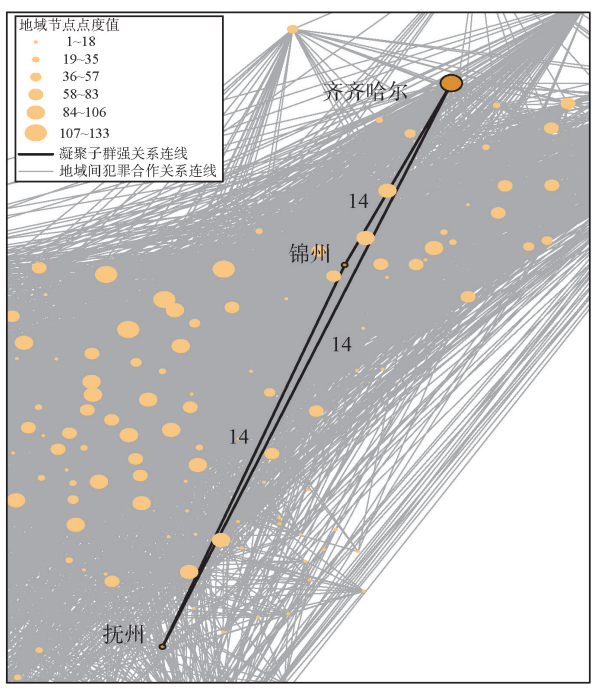

(e) 2014年子群一

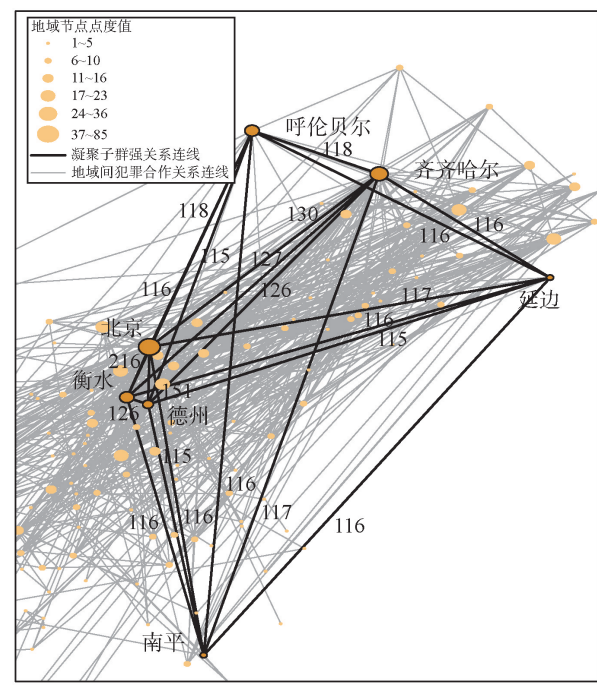

(b) 2005年子群二

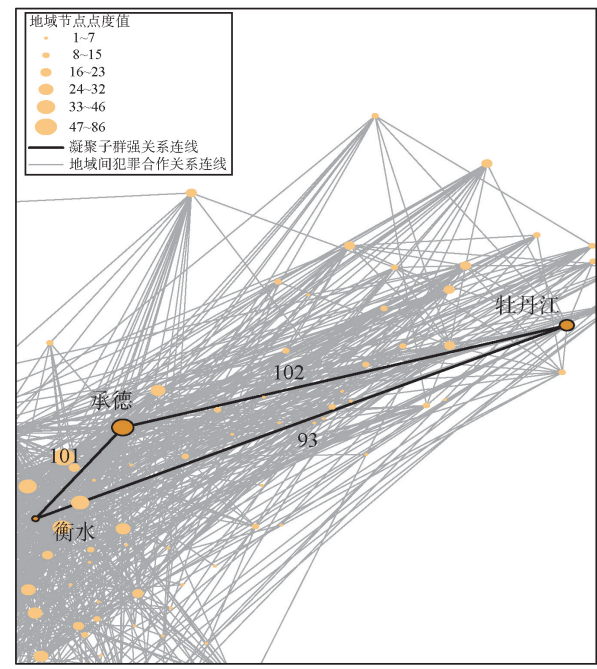

(d) 2010年子群二

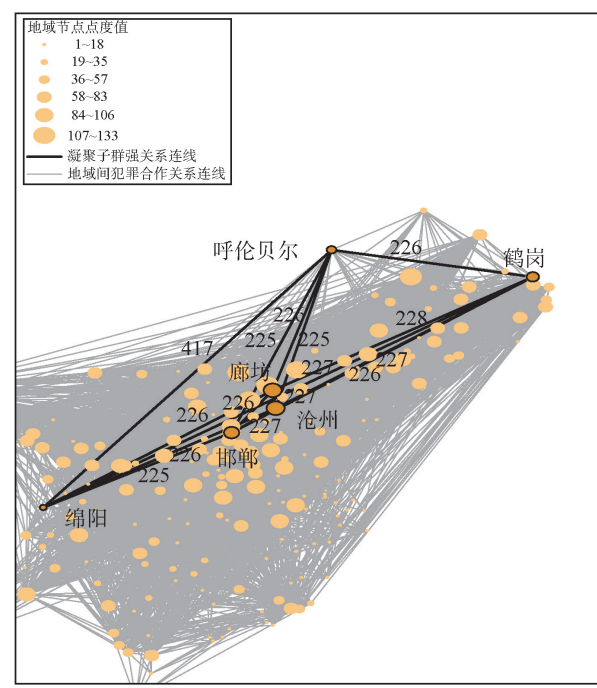

(f) 2014年子群二

图 5 基于共同犯罪的犯罪人地域关系网络高凝聚性子群空间结构

Fig.5 Spatial structures of high-cohesion subgroups of cross-area co-offending networks 
的重构 ${ }^{[42-43]}$ 。但是, 在外来人口重构社会关系及融 人城市的过程中, 往往会产生一定的社会问题。 首先, 在大城市环境中, 外来人口的经济水平普遍 较低, 因此会倾向于选择租金较低的区域居住, 这 就导致外来人口普遍聚居生活, 出现所谓的“城中 村” ${ }^{[44-45]}$; 其次, 外来人口往往仅具有初、高中文化程 度, 只能从事城市的底层行业, 其社会地位决定了 他们在城市中产生的社会关系仅局限于与其社会 地位相当的人群 ${ }^{[43]}$; 再次, 外来人口往往怀着发财 致富的梦想来到大城市, 但很多会因为劳资纠纷、 地域歧视、贫富差距等引起心理失衡, 因而渴望通 过较为强大的集体力量来满足自身需要 ${ }^{[46]}$ 。在这 种情况下, 相同的身份认同、生活诉求等将他们凝 聚起来, 并在这一群体内部形成一种“内卷化” 的认 同趋势, 从而形成外来人口中的犯罪亚文化 ${ }^{[4]}$ 。这 时, 当有人通过日常人际碰撞获取犯罪机会, 并进 而 “发家致富”之后, 其行为便产生示范效应, 并形 成犯罪行为传播, 从而在流动人群内产生共同犯罪 现象,并进而形成跨地域的犯罪人地域关系网络。

实际上, 通过之前的分析, 可以发现犯罪人地 域关系网络的基本模式在发生着改变, 而这一变化 过程也可以通过亚文化理论进行相应的解释。一 方面, 外来人口在进人新的城市后, 由于对城市的 社会环境不熟悉, 其犯罪行为的实施往往会受到现 实因素的制约, 而本地犯罪人群由于对当地环境相 对熟悉, 对作案地点选择、作案手段选取、销赃渠道 等更为了解, 因此, 外来人口极易在本地犯罪人群 的引领下实施犯罪行为。另一方面, 参与共同犯罪 的外来人口在通过犯罪行为获取到利益之后, 其行 为不仅会在当地外来人口群体内产生示范效应, 还 会造成其原籍地原有社会关系人员的不平衡心理, 这样, 在原籍地域性群体亚文化的作用下, 原籍的 社会关系人员被拉拢后便会倾向成为城市犯罪的 稳定主体供应来源 ${ }^{[48]}$ 。在本文针对北京市街头许 骗案件的研究中, 具体则表现为来自河北等地的犯 罪人群体通过其在北京市的社会关系重构不断发 展壮大, 其影响力不断提升甚至于超过当地犯罪人 群体, 从而使得北京市街头许骗犯罪人群体由地域 单中心模式向多中心模式转变。此外, 伴随着犯罪 人群体地域多中心模式的形成, 在外来人口内部逐 渐形成自发的犯罪牵引力, 加之北京到其他城市间 交通网络的日益完善、人口迁移成本的不断降低、 城市中的外来人口群体比重的增大, 外来人口的地
域文化多元性增强, 从而使来自不同地域的犯罪人 群之间的关系更加紧密, 导致原有的地域合作模式 被打破, 潜在犯罪者的地域性特征逐渐不再是合作 犯罪考虑的因素, 表现为各个地域之间的跨地域共 同犯罪现象愈加频繁 ${ }^{[49]}$ 。

\section{4 结论}

在共同犯罪现象中, 参与共同犯罪的犯罪人地 域属性特征是了解犯罪人关系模式的重要途径。 本文以北京市街头计骗案件为例, 利用犯罪人的地 理特征构建了基于共同犯罪的犯罪人地域关系网 络模型,对其2005、2010和2014年的网络结构特征 及其演变趋势进行了分析,发现 2005-2014年间北 京市街头计骗犯罪人群的地域来源数量增加, 但地 域的空间分布呈收缩态势, 来自各个地域的犯罪人 之间联系愈发紧密, 北京市街头计骗犯罪人群体逐 渐打破地域性的限制而倾向于成为一个有机整 体。此外, 随着犯罪人群体内部的不断整合, 其地 域关联模式也发生了改变,一方面体现在犯罪人群 的合作关系由以北京为核心的地域单中心型向以 河北等地为核心的地域多中心型转变; 另一方面表 现为犯罪人群中的地域性子群发生两极分化, 跨地 域性共同犯罪团伙的合作稳定性普遍下降。导致 这种现象存在及变化的原因是复杂的, 从犯罪文化 的角度, 可以认为在京外来人口因其特有的社会属 性而在社会关系重构过程中容易产生各种各样的 社会问题,进而在其群体内部形成了异于城市主流 价值观的犯罪亚文化,犯罪亚文化使不同地域外来 人口形成心理认同与犯罪主体供应, 同时日常活动 碰撞使犯罪人行为产生示范效应并形成了犯罪行 为传播, 从而导致了犯罪人地域关系网络的产生及 演变。

对基于共同犯罪的北京市街头计骗案件犯罪 人地域关系网络的结构特征及其变化过程进行分 析具有理论与实践两方面的意义。首先, 从理论 上, 通过对共同犯罪人地域关系网络及其变化进行 分析可以揭示出犯罪人群体的关系特征与形成过 程, 从而更加有效地发现亚文化在地域群体内部以 及外部进行传播与交互的过程及驱动机制, 这对于 更加全面地理解共同犯罪的产生原因具有很重要 的意义。其次, 从实践层面, 通过对共同犯罪人地 域关系模式进行分析,可以发现一些犯罪人潜在的 
合作偏好, 对这种模式的了解可以有助于进行犯罪 控制或犯罪预防。例如, 通过对网络节点影响力分 析可以发现“最受欢迎”的地域性犯罪人群,通过对 高影响力地域外来人口进行针对性排查与管控可 以对共同犯罪活动产生有效干扰; 通过对网络凝聚 子群进行挖掘可以探寻在犯罪活动中具有高并发 性的地域组合,通过对同行外来人口的地域伴随关 系进行合理监测可以有效实现犯罪预警。这些发 现都有助于公安机关根据外来人口的地域性特征 开展治安管理工作, 从而有效提升城市治理水平。

\section{参考文献(References)}

[1] Frank O. Statistical estimation of co-offending youth networks [J]. Social Networks, 2001, 23(3): 203-214.

[2] D"Alessio S J, Stolzenberg L. Do cities influence co- offending? [J]. Journal of Criminal Justice, 2010, 38(4): 711-719.

[3] Tayebi M A, Bakker L, Glasser U, et al. Locating central actors in co-offending networks [C]// International Conference on Advances in Social Networks Analysis \& Mining. Kaohsiung, China, 2011: 171-179.

[4] Lindquist M J, Zenou Y. Key players in co-offending networks [R]. IZA Discussion Paper No. 8012, 2014. https:// ssrn.com/abstract $=2409534$.

[5] Fischer C S. The subculture theory of urbanism: A twentieth-year assessment [J]. American Journal of Sociology, 1995, 101: 543-577.

[6] Mcgloin J M, Sullivan C J, Piquero A R, et al. Investigation the stability of co-offending and co-offenders among a sample of youthful offenders [J]. Criminology, 2010, 46 (1): 155-188.

[7] Grund T, Morselli C. Overlapping crime: Stability and specialization of co-offending relationships [J]. Social Network, 2017, 51: 14-22.

[8] Tillyer M S, Tillyer R. Co-offending, violence and situational moderators [J]. Journal of Criminal Justice, 2019, 64: $52-60$.

[9] Stevenson R J. The stolen goods market in New South Wales, Australia: An analysis of disposal avenues and tactics [J]. British Journal of Criminology, 2001, 41(1): 101-118.

[10] Antonopoulos G. Cigarette smuggling: A case study of a smuggling network in Greece [J]. European Journal of Crime, Criminal Law and Criminal Justice, 2006, 14(3): 239-255.

[11] Natarajan M. Understanding the structure of a large heroin distribution network: A quantitative analysis of qualitative data [J]. Journal of Quantitative Criminology, 2006,
22(2): 171-192.

[12] Kersel M M. Transcending borders: Objects on the move [J]. Archaeologies, 2007, 3(2): 81-98.

[13] Coluccello S, Massey S. Out of Africa: The human trade between Libya and Lampedusa [J]. Trends in Organized Crime, 2007, 10(4): 77-90.

[14] Malm A, Bichler G, Stephanie V D W. Comparing the ties that bind criminal networks: Is blood thicker than water? [J]. Security Journal, 2010, 23(1): 52-74.

[15] Schaefer D R. Youth co-offending networks: An investigation of social and spatial effects [J]. Social Network, 2012, 34: 141-149.

[16] Charette Y, Papachristos A V. The network dynamics of co-offending careers [J]. Social Network, 2017, 51: 3-13.

[17] Zhang S X, Chin K L, Miller J. Women's participation in Chinese transnational human muggling: A gendered market perspective [J]. Criminology, 2007, 45(3): 699-733.

[18] Godson R, Olson W J. International organized crime[J]. Society, 1995, 32(2): 18-29.

[19] Mcillwain J S. Organized crime: A social network approach [J]. Crime Law \& Social Change, 1999, 32(4): 301-323.

[20] Tenti V, Morselli C. Group co-offending networks in Italy's illegal drug trade $[\mathrm{J}]$. Crime, Law and Social Change, 2014, 62(1): 21-44.

[21] O'Neill A. International trafficking in women from Central Europe and the NIS [J]. Trends in Organized Crime, 1998, 4(2): 133-136.

[22] Albanese J. White-collar crime in America [M]. Englewood Cliffs, USA: Prentice Hall, 1995.

[23] 白晓荣. 兰州市东乡族外来人口聚落的社会关系研究 [J]. 中南民族大学学报(人文社会科学版), 2013, 33(2): 71-74. [Bai Xiaorong. Study on the social relationship of Dongxiang floating population in Lanzhou. Journal of South- Central University for Nationalities (Humanities and Social Sciences), 2013, 33(2): 71-74. ]

[24] 李吉和, 杨春娥. 中、东部地区城市穆斯林外来人口社 会关系融人状况: 基于武汉、广州、杭州、宁波的调查 [J]. 西南民族大学学报(人文社科版), 2015, 36(5): 1923. [Li Jihe, Yang Chun'e. Integration of social relations among urban Muslim migrants in central and eastern China: Based on a survey in Wuhan, Guangzhou, Hangzhou and Ningbo. Journal of Southwest Minzu University (Humanities and Social Science), 2015, 36(5): 19-23. ]

[25] 陈鹏, 胡啸峰, 篗珂. 基于社会网络分析的犯罪人团伙 地域关系研究 [J]. 中国刑警学院学报, 2016(2): 3-6. [Chen Peng, Hu Xiaofeng, Qu Ke. Research on the regional relationship of criminal groups based on social 
network analysis. Journal of Criminal Investigation Police University of China, 2016(2): 3-6. ]

[26] Chen P, Lu Y. Exploring co-offending networks by considering geographic background: An investigation of electric bicycle thefts in Beijing [J]. The Professional Geographer, 2018, 70(1): 73-83.

[27] 李丹丹, 汪涛, 周辉. 基于不同时空尺度的知识溢出网 络结构特征研究 [J]. 地理科学, 2013, 33(10): 11801187. [Li Dandan, Wang Tao, Zhou Hui. The structural characteristics of knowledge spillover networks based on different spatial and temporal scales. Scientia Geographica Sinica, 2013, 33(10): 1180-1187. ]

[28] 刘承良, 管明明, 段德忠. 中国城际技术转移网络的空 间格局及影响因素 [J]. 地理学报, 2018, 73(8): 70-85. [Liu Chengliang, Guan Mingming, Duan Dezhong. Spatial pattern and influential mechanism of interurban technology transfer network in China. Acta Geographica Sinica, 2018, 73(8): 70-85. ]

[29] 于谨凯, 马健秋. 山东半岛城市群经济联系空间格局演 变研究 [J]. 地理科学, 2018, 38(11): 1875-1882. [Yu Jinkai, Ma Jianqiu. Spatial pattern evolution of economic links of urban agglomeration in Shandong Peninsula. Scientia Geographica Sinica, 2018, 38(11): 1875-1882. ]

[30] 刘承良,牛彩澄. 东北三省城际技术转移网络的空间演 化及影响因素 [J]. 地理学报, 2019, 74(10): 2092-2107. [Liu Chengliang, Niu Caicheng. Spatial evolution and factors of interurban technology transfer network in Northeast China from national to local perspectives. Acta Geographica Sinica, 2019, 74(10): 2092-2107. ]

[31] 赵金丽, 盛彦文, 张璐璐, 等. 基于细分行业的中国城市 群金融网络演化 [J]. 地理学报, 2019, 74(4): 109-122. [Zhao Jinli, Sheng Yanwen, Zhang Lulu, et al. Evolution of urban agglomeration financial network in China based on subdivision industry. Acta Geographica Sinica, 2019, 74(4): 109-122. ]

[32] 马佳卉, 贺灿飞. 中间产品贸易网络结构及其演化的影 响因素探究: 基于贸易成本视角 [J]. 地理科学进展, 2019, 38(10): 1607-1620. [Ma Jiahui, He Canfei. Structure and change of international trade network of intermediate goods: From the perspective of trade costs. Progress in Geography, 2019, 38(10): 1607-1620. ]

[33] Liu C, Xu J, Zhang H. Competitiveness or complementarity? A dynamic network analysis of international agritrade along the belt and road [J]. Applied Spatial Analysis and Policy, 2019. doi: 10.1007/s12061-019-09307-5.

[34] 任怡. 街头计骗案件的分析与控制对策 [J]. 北京警察 学院学报, 2006(4): 46-48. [Ren Yi. Analysis and control countermeasures of street fraud cases. Journal of Beijing
Police College, 2006(4): 46-48. ]

[35] 汤放华, 汤迪莎, 汤慧, 等. 长江中游城市集群经济网络 结构分析 [J]. 地理学报, 2013, 68(10): 1357-1366. [Tang Fanghua, Tang Disha, Tang Hui, et al. Analysis of the economic network structure of urban agglomerations in the middle Yangtze River. Acta Geographica Sinica, 2013, 68(10): 1357-1366. ]

[36] 刘军. 整体网分析讲义: UCINET 软件实用指南[M].上 海: 格致出版社, 2009. [Liu Jun. Lecture on whole network approach: A practical guide to UCINET. Shanghai, China: Truth \& Wisdom Press, 2009. ]

[37] 德・诺伊, 安德烈·姆尔, 弗拉迪米尔・巴塔盖尔. 蜘蛛: 社会网络分析技术 [M]. 林枫, 译. 北京: 世界图书出版 公司, 2014. [NooyW, Mrvar A, Batagelj V. Pajek: Social network analysis technology. Translated by Lin Feng. Beijing, China: World Publishing Corporation, 2014. ]

[38] 吴康, 方创琳, 赵泪少. 中国城市网络的空间组织及其 复杂性结构特征 [J]. 地理研究, 2015, 34(4): 711-728. [Wu Kang, Fang Chuanglin, Zhao Miaoxi. The spatial organization and structure complexity of Chinese intercity networks. Geographical Research, 2015, 34(4): 711-728. ]

[39] Freeman L C. Centrality in social networks' conceptual clarification [J]. Social Networks, 1979, 1(3): 215-239.

[40] 王诺, 董玲玲, 吴暖, 等. 蓄意攻击下全球集装箱海运网 络脆弱性变化 [J]. 地理学报, 2016, 71(2): 293-303. [Wang Nuo, Dong Lingling, Wu Nuan, et al. The change of global container shipping network vulnerability under intentional attack. Acta Geographica Sinica, 2016, 71(2): 293-303. ]

[41] 龙冬平,柳林,周素红,等. 地理学视角下犯罪者行为研究 进展 [J]. 地理科学进展, 2017, 36(7): 886-902. [Long Dongping, Liu Lin, Zhou Suhong, et al. Research progress of criminal behavior from the perspective of geography. Progress in Geography, 2017, 36(7): 886-902. ]

[42] 严小兵, 焦华富. 犯罪地理国际研究进展 [J]. 地理科学 进展，2012，31(10)：1390- 1398. [Yan Xiaobing，Jiao Huafu. A review on the foreign criminal geography research. Progress in Geography, 2012, 31(10): 13901398. ]

[43] 叶鹏飞, 康熙熙, 王鼎. 流动青年的关系重构与城市融 人: 北京市 $\mathrm{F}$ 社区青年汇的案例分析 [J]. 北京青年研 究, 2015(1): 66-72. [Ye Pengfei, Kang Xixi, Wang Ding. Relationship reconstruction and urban integration of floating youth: A case study about F community youth gathering in Beijing. Beijing Youth Research, 2015(1): 66-72.]

[44] 任义科, 宋连成, 余瑞芳, 等. 属性和网络结构双重视角 下农民工流动规律研究 [J]. 地理科学进展, 2017, 36 
(8): 940-951. [Ren Yike, Song Liancheng, Yu Ruifang, et al. Migrant workers' migration patterns from the dual perspectives of attributes and network structures. Progress in Geography, 2017, 36(8): 940-951. ]

[45] 姚乐. 城中村外来人口社会融人研究 [J]. 轻工科技, 2019(6): 138-139. [Yao Le. Research on social integration of floating population in urban villages. Guangxi Journal of Light Industry, 2019(6): 138-139. ]

[46] 李卒. 亚文化与广州市外来人口犯罪 [J]. 广州市公安 管理干部学院学报, 2001(4): 4-12. [Li Zu. Subculture and crime of floating population in Guangzhou. Journal of Guangzhou Police College, 2001(4): 4-12. ]

[47] 叶鹏飞. 探索农民工城市社会融合之路: 基于社会交往 “内卷化”的分析 [J]. 城市发展研究, 2012(1): 89-93, 117. [Ye Pengfei. Exploring the road of urban social inte- gration of migrant workers: Based on the analysis of integration about social interaction. Urban Studies, 2012(1): 89-93, 117. ]

[48] 任九光. 地域性犯罪群体的概念、成因及预防: 对北京 地区外来人口犯罪状况的考察 [J]. 中国人民公安大学 学报, 2004(5): 149-154. [Ren Jiuguang. The concept, cause and prevention of regional crime group: Investigation on the crime situation of floating population in Beijing. Journal of Chinese People's Public Security University (Social Sciences Edition), 2004(5): 149-154. ]

[49] 王明涵, 于莉. 城市化背景下外来人口的城市融人研究 [J]. 管理观察, 2019(8): 89-91, 97. [Wang Minghan, Yu Li. Urban integration of floating population under the background of urbanization. Management Observer, 2019 (8): 89-91, 97. ]

\title{
Spatial evolution and influencing factors of criminals' cross-area co-offending network: An example of fraud in Beijing
}

\author{
ZHU Guanyu, CHEN Peng* \\ (School of Information Engineering and Cyber Security, People's Public Security University of China, Beijing 100038, China)
}

\begin{abstract}
A series of studies have been conducted on the phenomenon of co-offending from the aspects of relationship and relationship structure of offenders, but there is a lack of stability analysis on the regional relationship between offenders. Based on the data of the street fraud cases in Beijing in 2005, 2010, and 2014, this study constructed a regional relation network model of offenders based on the principle of social network, and analyzed the structural characteristics and changing trend of the regional relation network of offenders participating in co- offending by means of network analysis. The main results are as follows. The spatial distribution of origins of offenders participating in the co-offending in Beijing was gradually concentrated, and a pattern of coexistenting multicenters was formed with North China as the main area. The small-world effect of regional relation network of offenders gradually strengthened and developed from power-law distribution mode to exponential distribution mode. Among the offenders who participated in the co-offending, the influence of offenders from Beijing gradually decreased, while that of offenders from Hebei Province gradually increased. The cohesive subgroup structure of the regional relation network of offenders gradually polarized, and a few offender regional subgroup structures that are closely related to co-offending appeared. This study also explored the reasons for the existence and evolution of the cross-area co-offending network from the perspectives of social relation reconstruction and subculture of floating population. The results of this study have some implications for the further research on the relationship pattern of the offenders' co-offending.
\end{abstract}

Keywords: fraud crime; regional relationship network; comparative analysis of changes; social network analysis; Beijing 